ISSN 1561-8358 (Print)

ISSN 2524-244X (Online)

\author{
ЭНЕРГЕТИКА, ТЕПЛО- И МАССООБМЕН \\ POWER ENGINEERING, HEAT AND MASS TRANSFER
}

УДК 621.311

https://doi.org/10.29235/1561-8358-2019-64-4-438-446

Поступила в редакцию 12.07.2019

Received 12.07.2019

\author{
А.С. Мельников ${ }^{1}$, Б. И. Попов ${ }^{2}$ \\ ${ }^{1}$ Объединенный институт энергетических и ядерных исследований-Сосны Нацииональной академии наук \\ Беларуси, Минск, Беларусь \\ ${ }^{2}$ Институт энергетики Национальной академии наук Беларуси, Минск, Беларусь

\section{ОПРЕДЕЛЕНИЕ ЭФФЕКТИВНОСТИ КОНДЕНСАЦИОННЫХ И ТЕПЛОФИКАЦИОННЫХ ТУРБОАГРЕГАТОВ НА ОСНОВЕ ЭНЕРГЕТИЧЕСКИХ ХАРАКТЕРИСТИК ТУРБИН}

\begin{abstract}
Аннотация. Решалась задача расчета коэффициентов полезного действия турбоагрегатов различного типа, входящих в состав оборудования источников электрической и тепловой энергии. В упрощенных подходах к представлению турбоагрегатов, часто используемых в алгоритмах программ оптимизации энергосистем, в целях сокращения расчетного времени игнорируется зависимость эффективности установки от текущей мощности, не учитывается влияние величины давления в отборах пара теплофикационных турбин для покрытия тепловой нагрузки и наличие холостого хода турбин. Указанные подходы просты в использовании и реализации, однако могут вносить заметные погрешности в результаты оптимизации ввиду отсутствия учета параметров и зависимостей, имеющих место в реальных режимах работы оборудования и значительно влияющих на количество используемого оптимизируемой системой топлива. В отличие от упрощенного подхода, в работе для определения коэффициентов полезного действия, характеризующих эффективность турбоагрегатов, использованы энергетические характеристики. Это позволяет давать более точную оценку эффективности в различных режимах работы без существенного усложнения алгоритма с незначительным увеличением расчетного времени. Использование предлагаемого подхода показало наличие заметного уточнения эффективности по сравнению с простыми моделями и, следовательно, целесообразность представления турбоагрегатов с использованием энергетических характеристик в алгоритмах программ, предназначенных для оптимизации энергосистем.

Ключевые слова: коэффициент полезного действия, турбоагрегат, эффективность, энергетические характеристики, алгоритм, программа, оптимизация, энергосистема

Для цитирования: Мельников, А.С. Определение эффективности конденсационных и теплофикационных турбоагрегатов на основе энергетических характеристик турбин / А. С. Мельников, Б. И. Попов // Вес. Нац. акад. навук Беларусі. Сер. фіз.-тэхн. навук. - 2019. - Т. 64, №4. - С. 438-446. https://doi.org/10.29235/1561-8358-2019-64-4-438-446
\end{abstract}

\author{
A.S. Melnikov' ${ }^{1}$ B. I. Popov² \\ ${ }^{1}$ Joint Institute for Power and Nuclear Research - Sosny of the National Academy of Sciences of Belarus, Minsk, Belarus \\ ${ }^{2}$ Institute of Energy of the National Academy of Sciences of Belarus, Minsk, Belarus
}

\title{
DETERMINATION OF EFFICIENCY OF CONDENSATION AND HEAT TEFLIFICATION TURBO UNITS ON THE BASIS OF TURBINE ENERGY CHARACTERISTICS
}

Abstract. In this work, the problem of calculating the efficiency of turbo-units of various types that are part of the equip-
ment of electric and thermal energy sources was solved. In simplified approaches to the representation of turbine units, often
used in algorithms of power system optimization programs, in order to reduce the estimated time, the dependence of the plant
efficiency on the current power is ignored, the influence of the pressure in the steam extraction of cogeneration turbines to cover
the heat load and the idle speed of the turbines are not taken into account. These approaches are easy to use and implement, how-
ever, they can introduce noticeable errors in the optimization results due to the lack of consideration of parameters and depen-
dencies that occur in real equipment operation modes and significantly affect the amount of fuel used by the optimized system.
In contrast to the simplified approach, energy characteristics were used in the work to determine the efficiency factors character-

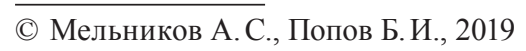


izing the efficiency of turbine units. This allows providing a more accurate assessment of the effectiveness in various operating modes without significantly complicating the algorithm with a slight increase in the estimated time. Using the proposed approach showed the presence of a noticeable refinement of efficiency compared to simple models and, therefore, the feasibility of representing turbine units using energy characteristics in program algorithms designed to optimize energy systems.

Keywords: efficiency, turbine unit, efficiency, energy characteristics, algorithm, program, optimization, power system

For citation: Melnikov A. S., Popov B. I. Determination of efficiency of condensation and heat teflification turbo units on the basis of turbine energy characteristics. Vestsi Natsyyanal'nai akademii navuk Belarusi. Seryya fizika-technichnych navuk = Proceedings of the National Academy of Sciences of Belarus. Physical-technical series, 2019, vol. 64, no. 4, pp. 438-446 (in Russian). https://doi.org/10.29235/1561-8358-2019-64-4-438-446

Введение. Инструменты оптимизации энергетических систем нуждаются в простых алгоритмах расчета эффективности турбоагрегатов различного типа для производства электрической и тепловой энергии.

В алгоритмах оптимизационных программ (см., например, [1]) эффективность работы турбины обычно характеризуется коэффициентом полезного действия (КПД), который определяется при номинальной мощности и не зависит от ее текущего значения. Простота таких алгоритмов позволяет использовать их без больших затрат машинного времени. Однако такой подход имеет ряд недостатков, самым принципиальным из которых является игнорирование зависимости эффективности работы турбин от уровня мощности, хотя в действительности эта зависимость может быть весьма существенной. На эффективность работы теплофикационных турбин оказывает также влияние величина давления в отборах пара потребителю тепла. Наконец, такие алгоритмы не учитывают наличие холостого хода турбин.

Указанные недостатки могут быть в значительной степени устранены без большого увеличения сложности расчетного алгоритма использованием для определения эффективности турбоагрегатов энергетических характеристик.

Энергетическая характеристика представляет собой зависимость расхода тепла на турбину от электрической мощности турбоагрегата и мощности регулируемых отборов пара $[2,3]$. Энергетические характеристики могут быть представлены в различном виде, в частности как типовые, носящие нормативный характер и включающие диаграммы режимов с поправками для оценок показателей турбоагрегатов [3]. Однако, имея в виду дальнейшую разработку алгоритмов оптимизационных программ, значительно удобнее иметь аналитические выражения для энергетических характеристик. Поэтому далее используем аналитические выражения энергетических характеристик для ряда турбин, взятые из [2].

Турбоагрегат с конденсационной турбиной. Эффективность работы турбоагрегата с конденсационной турбиной можно охарактеризовать коэффициентом полезного действия (КПД) $\eta_{t u}$ :

$$
\eta_{t u}=\frac{N}{Q_{0}},
$$

где $N$ - электрическая мощность генератора, МВт; $Q_{0}$ - энергетическая характеристика турбоагрегата, которая дает расход тепла на турбину, обеспечивающий данную мощность, МВт.

Энергетическая характеристика турбоагрегата с конденсационной турбиной $Q_{0}$ рассчитывается по формуле [2]

$$
Q_{0}=Q_{i d}+r N,
$$

где $Q_{i d}$ - расход тепла на холостой ход турбины (при нулевой нагрузке), $\mathrm{MBT} ; r$ - относительный прирост количества тепла.

Подставляя (2) в (1), получаем

$$
\eta_{t u}=\frac{N}{Q_{i d}+r N}=\frac{1}{\frac{Q_{i d}}{N}+r} .
$$

Таким образом, выражение (3) определяет зависимость КПД от мощности.

Чтобы оценить, насколько велика может быть погрешность представления турбоагрегата единственным значением КПД на номинальной мощности, рассмотрим в качестве примера энергетическую характеристику турбоагрегата К-300-240, которая рассчитывается следующим образом [2]:

$$
Q_{0}=50+1,967 N \text {. }
$$


Зависимости рассчитанных по выражениям (2) и (3) энергетической характеристики и КПД турбоагрегата К-300-240 от мощности показаны на рис. 1.
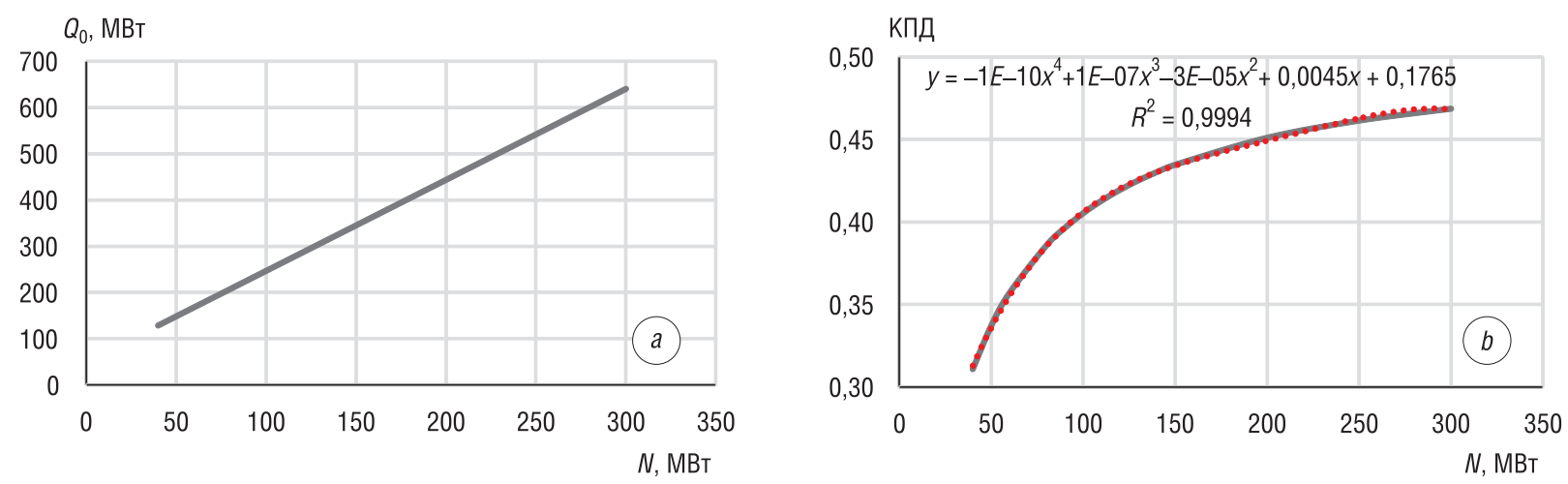

Рис. 1. Зависимости энергетической характеристики и КПД турбоагрегата К-300-240 от мощности

Fig. 1. Dependences of the energy characteristics and efficiency of the K-300-240 turbine unit on power

КПД турбоагрегата, как видно из выражения на рис. $1, b$, с высокой степенью достоверности представляется полиномом 4-го порядка, что подтверждается близостью к единице статистического параметра $R^{2}$, характеризующего качество представления. В этом выражении $x$ - значение мощности, $y$ - значение КПД. В алгоритме оптимизационной программы, таким образом, можно с одинаковым успехом использовать как прямой расчет КПД по выражению (3), так и аппроксимацию, показанную красными точками на рис. $1, b$.

Как видно из рис. $1, b$, КПД имеет ярко выраженную нелинейность при мощности, меньшей $50 \%$ от номинальной. При мощности $50 \%$ от номинальной, значения КПД меньше более чем на $7 \%$ относительных (по отношению к КПД на номинальной мощности), а при мощности $30 \%$ от номинала - на 13,5 \%. Такая существенная зависимость КПД от мощности требует учета при проведении оптимизационных расчетов или, по крайней мере, анализа необходимости такого учета.

Турбоагрегаты с теплофикационными турбинами. В общем виде энергетическая характеристика турбоагрегата с производственным и отопительным отборами пара представляется в виде [2]

$$
\begin{gathered}
Q_{0}=Q_{i d}+r_{k} N-\left(r_{k}-r_{t}\right) N_{t}+\sum Q_{h}, \\
N_{t}=W_{i n}^{p} Q_{h}^{p}+W_{i n}^{h} Q_{h}^{h}-\Delta N_{i d},
\end{gathered}
$$

где $r_{k}, r_{t}$ - удельный расход тепла для конденсационного и теплофикационного потоков пара соответственно; $\sum Q_{h}=Q_{h}^{p}+Q_{h}^{h}$ - полная тепловая нагрузка производственного и отопительного отборов, МВт; $W_{i n}^{p}, W_{i n}^{h}$ - удельная выработка электроэнергии на тепловом потреблении для производственного и отопительного отборов соответственно; $\Delta N_{i d}$ - условная мощность холостого хода, МВт; $N_{t}$ - мощность на тепловом потреблении, МВт.

Используем (4) и (5) для определения эффективности турбины в частных случаях - для турбины противодавления типа Р и теплофикационной типа Т.

Tурбоагрегат с теплофикационной турбиной типа $\boldsymbol{P}$. Для турбоагрегата этого типа $Q_{h}^{h}=0$ и $N=N_{t}$, поэтому выражения (4) и (5) приобретают вид:

$$
\begin{gathered}
Q_{0}=Q_{i d}+r_{t} N_{t}+Q_{h}^{p}, \\
N_{t}=W_{i n}^{p} Q_{h}^{p}-\Delta N_{i d}, \\
W_{i n}^{p}=a\left(\frac{P_{n}}{p_{p}}\right)^{C}, \\
\Delta N_{i d}=a b\left(\frac{p_{n}}{p_{p}}\right)^{C},
\end{gathered}
$$


где мощность холостого хода $Q_{i d}$ зависит только от типа турбины; $a, b$ и показатель степени $C-$ известные величины, также зависящие от типа турбины; $p_{n}$ и $p_{p}$ - соответственно номинальное и текущее значения давления в производственном отборе турбины.

Из (6) видно, что поток тепла, поступающего в турбину, можно условно разделить на потоки холостого хода, производства электроэнергии (на тепловом потреблении) и производства тепла (для внешнего потребителя). Поэтому в соответствии с таким разделением эффективность работы турбоагрегата можно охарактеризовать следующими условными составляющими КПД:

КПД по производству электроэнергии $\eta_{e}$ :

КПД по производству тепла $\eta_{h}$ :

$$
\eta_{e}=\frac{N_{t}}{Q_{0}}
$$

полный КПД ๆ:

$$
\eta_{h}=\frac{Q_{h}^{p}}{Q_{0}}
$$

$$
\eta=\eta_{e}+\eta_{h}
$$

В отличие от простого подхода, при котором турбина характеризуется номинальными значениями КПД по производству электроэнергии и тепла во всем диапазоне мощностей, использование энергетической характеристики позволит учитывать зависимость составляющих КПД от таких параметров, как уровень электрической и тепловой мощности и противодавление.

В качестве численного примера рассмотрим модификацию турбины P-50-130/13 [4], основные показатели которой приведены в табл. 1.

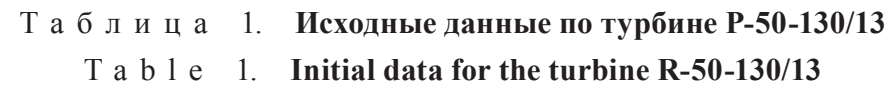

\begin{tabular}{|l|c|}
\hline \multicolumn{1}{|c|}{ Параметр } & 3начение \\
\hline Электрическая мощность номинальная/максимальная, МВт & $52,7 / 60$ \\
\hline Номинальное противодавление, МПа & 1,275 \\
\hline Номинальная тепловая мощность*, МВт & 224 \\
\hline Удельный расход тепла для теплофикационного потока пара, МВт/МВт & 1,01 \\
\hline Расход тепла на холостой ход, МВт & 1,164 \\
\hline Коэффициент $a$ & 0,3 \\
\hline Величина $b$, МВт & 48,3 \\
\hline Показатель степени $C$ & 0,55 \\
\hline
\end{tabular}

*Рассчитана по выражению (6) при номинальной электрической мощности и номинальном противодавлении.

В табл. 1 приведены также коэффициенты для расчета энергетической характеристики, входящие в формулы (6)-(9).

Электрическая мощность на тепловом потреблении $N_{t}$, как видно из выражений (7)-(9), зависит от величины противодавления $p_{T}$. Характер этой зависимости при номинальной тепловой мощности показан на рис. 2.

Как видно из формулы на рисунке, зависимость хорошо описывается полиномиальной зависимостью 3-й степени. В формуле $x$ - значение противодавления, $y$ - значение электрической мощности. В области номинального значения противодавления уменьшение его значения на 14 \% приводит к увеличению электрической мощности примерно на 8 \%. Такая достаточно сильная зависимость требует учета при проведении оптимизационных расчетов.

На рис. 3 показана зависимость электрической мощности от тепловой, полученная с помощью формул (6)-(12). Из выражения (7) понятно, что электрическая и тепловая мощности турбоагрегата связаны линейной зависимостью. Учет холостого хода турбины приводит к тому, что электрическая мощность равна 0 до определенного значения тепловой мощности, равного 48,3 МВт для турбины типа Р. 
442 Proceedings of the National Academy of Sciences of Belarus. Physical-technical series, 2019, vol. 64, no. 4, pp. 438-446

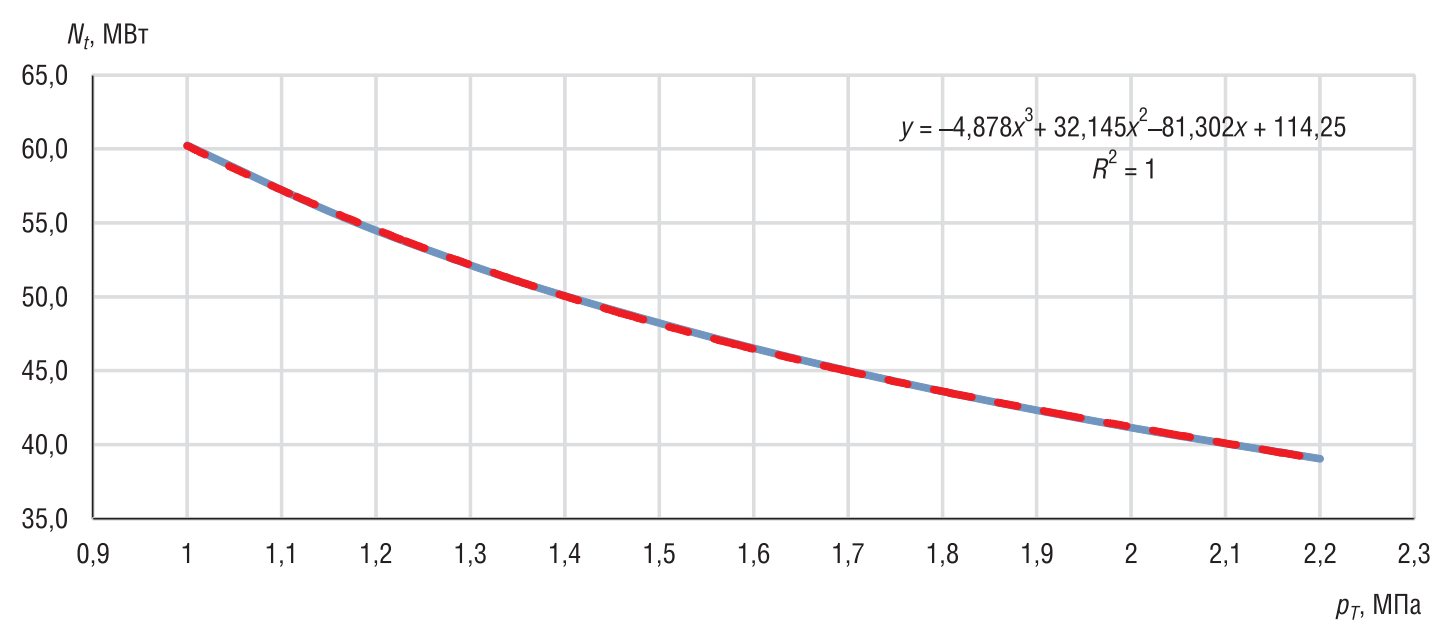

Рис. 2. Зависимость электрической мощности от противодавления при номинальной тепловой мощности

Fig. 2. Dependence of electric power on backpressure at rated thermal power

На этом же рисунке показана зависимость, используемая при простом подходе [1]. Поскольку в данном подходе не учитывается наличие холостого хода турбины, линейная зависимость связывает нулевые и номинальные значения электрической и тепловой мощности.

Как видно, простой подход завышает величину электрической мощности, которую можно получить при определенной тепловой мощности. При тепловой мощности около 40 \% от номинальной превышение составит около 50 \% от более корректного значения, полученного с использованием энергетической характеристики. Такая погрешность может оказать существенное влияние на результаты оптимизационных расчетов.

На рис. 4 представлены составляющие и полный КПД, рассчитанные по формулам (10)-(12), также в зависимости от тепловой мощности.

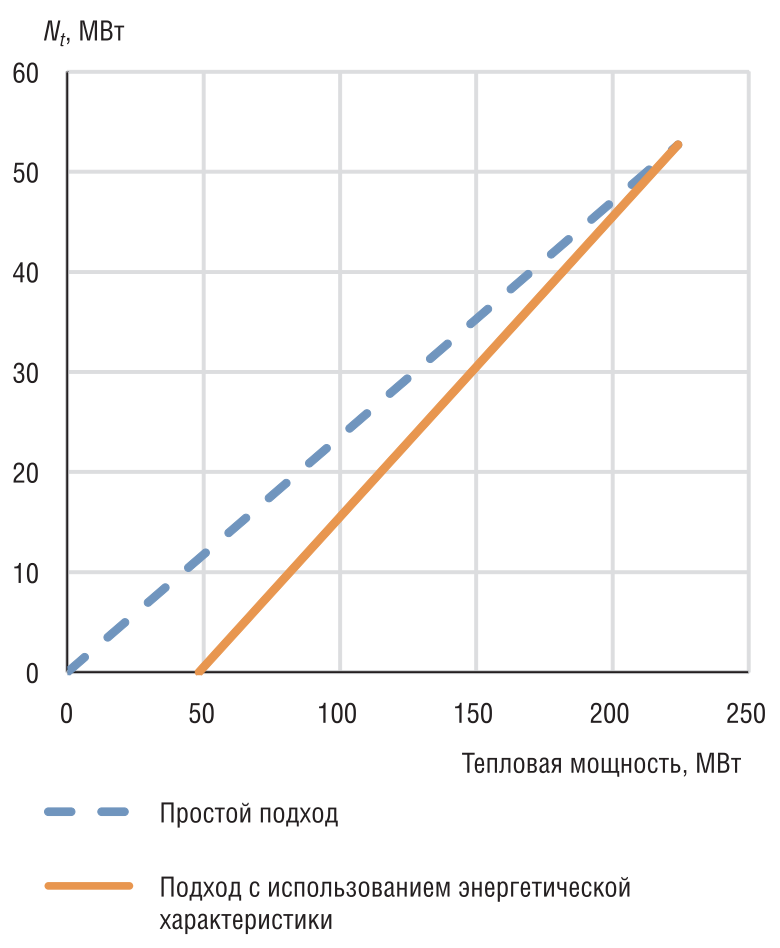

Рис. 3. Соотношения тепловой и электрической мощности, полученные при простом подходе и с использованием энергетической характеристики

Fig. 3. Ratios of thermal and electric power obtained with a simple approach and using of the energy characteristics

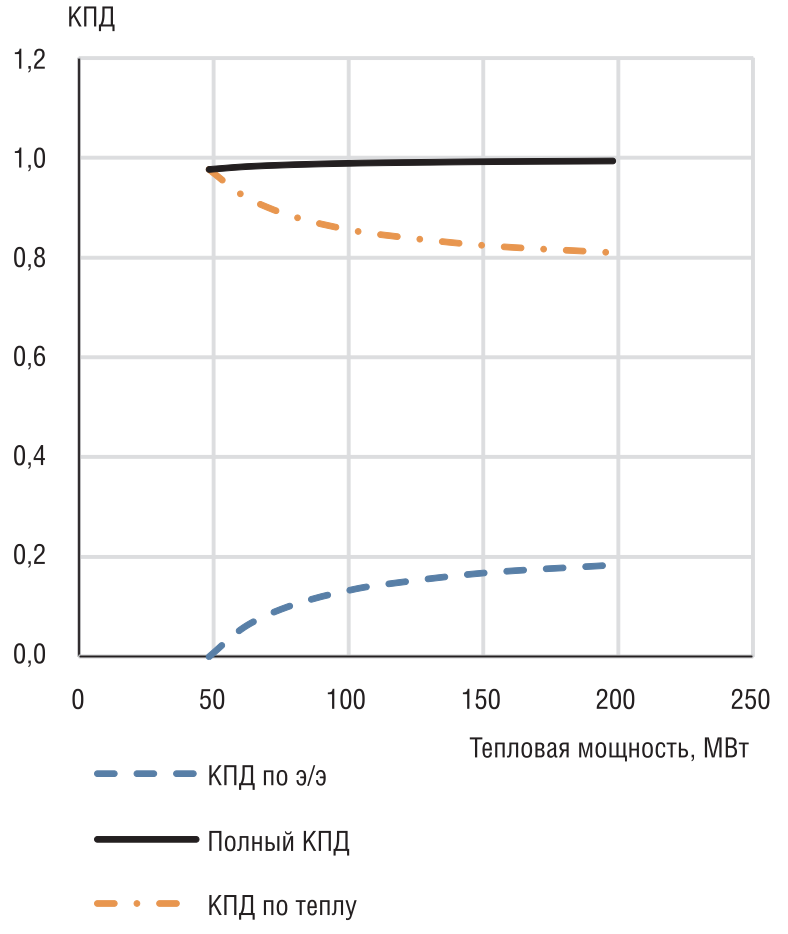

Рис. 4. Зависимость КПД турбины Р-50-130/13 от тепловой мощности

Fig. 4. The dependence of the efficiency of the turbine R-50$130 / 13$ on thermal power 
Полный КПД, как видно из рис. 4 , изменяется незначительно, отражая противоположный ход зависимостей его составляющих по электроэнергии и по теплу. Однако составляющая по электроэнергии меняется весьма существенно. Так, при мощности в $40 \%$ от номинальной КПД по электроэнергии составляет менее $60 \%$ от его номинального значения. Это также может сказываться на результатах оптимизационных расчетов и говорит о целесообразности оценки эффективности турбоагрегатов с применением энергетических характеристик.

Tурбоагрегат с теплофикационной турбиной типа $T$. Поскольку турбины этого типа имеют только отопительные отборы пара, выражения (4) и (5) преобразуются:

$$
\begin{gathered}
Q_{0}=Q_{i d}+r_{k} N-\left(r_{k}-r_{t}\right) N_{t}+Q_{h}^{h}=Q_{i d}+r_{k} N_{k}+r_{t} N_{t}+Q_{h}^{h}, \\
N_{t}=W_{i n}^{h} Q_{h}^{h}-\Delta N_{i d}, \\
W_{i n}^{h}=e Q_{h}^{h} p_{T}^{-F}, \\
\Delta N_{i d}=g p_{T}^{-F},
\end{gathered}
$$

где $Q_{i d}=d p_{T} ; N_{k}=N-N_{t}$ - конденсационная мощность турбоагрегата; $d, e, g$ и показатель степени $F$ - величины, зависящие от типа турбины.

Для турбоагрегатов данного типа можно ввести понятия следующих условных составляющих КПД:

КПД по производству электроэнергии конденсационным потоком пара:

$$
\eta_{e}^{K}=\frac{N_{k}}{Q_{0}}
$$

КПД по производству электроэнергии теплофикационным потоком пара:

КПД по производству тепла:

$$
\eta_{e}^{T}=\frac{N_{t}}{Q_{0}}
$$

$$
\eta_{h}=\frac{Q_{h}^{h}}{Q_{0}},
$$

полный КПД турбоагрегата:

$$
\eta=\eta_{e}^{K}+\eta_{e}^{T}+\eta_{h}
$$

В качестве численного примера рассмотрим турбину Т-175/210-130 [3]. Основные характеристики турбины приведены в табл. 2.

В табл. 3 приведены значения коэффициентов, рассчитанные по формулам (14)-(16).

Используя зависимости (13)-(16), определим, что номинальное значение электрической мощности 175 МВт при максимальной тепловой нагрузке 325 МВт реализуется при давлении в верхнем отборе турбины 0,124 МПа. Это значение давления примем за номинальное и для него проведем дальнейшие выкладки. По формуле (13) можно также подсчитать максимальную электрическую мощность турбины, реализуемую в конденсационном режиме работы. Эта мощность оказывается равной 206 МВт и отличается от значения 210 МВт, приведенного в табл. 2, менее чем на $2 \%$. Таким образом, выражение для энергетической характеристики адекватно воспроизводит основные характеристики турбины.

Т а б л и ц а 2. Характеристики турбины T-175/210-130

$\mathrm{T} \mathrm{a} \mathrm{b} 1 \mathrm{e} 2$. Characteristics of the turbine T-175/210-130

\begin{tabular}{|l|c|}
\hline \multicolumn{1}{|c|}{ Наименование характеристики } & Значение \\
\hline Мощность, МВт & 175 \\
номинальная & 210 \\
максимальная & 325 \\
\hline Максимальная тепловая нагрузка, МВт & $0,05-0,29$ \\
\hline Диапазон давления в верхнем отборе, МПа & 0
\end{tabular}

Т а б л и ц а 3. Коэффициенты для расчета энергетической характеристики турбины T-175/210-130

$\mathrm{T}$ a b 1 e 3. The coefficients for calculation of energy characteristic for the turbine T-175/210-130

\begin{tabular}{|c|c|}
\hline $\begin{array}{c}\text { Наименование } \\
\text { коэффициента }\end{array}$ & Значение \\
\hline$r_{k}$ & 2,316 \\
\hline$r_{t}$ & 1,016 \\
\hline$d$ & 198,9 \\
\hline$e$ & 0,433 \\
\hline$F$ & $-0,140$ \\
\hline$g$ & 10,576 \\
\hline
\end{tabular}


С учетом выражений (13)-(16), построены границы области работы турбины типа Т (рис. 5), внутри которой возможны произвольные соотношения электрической и тепловой мощности.

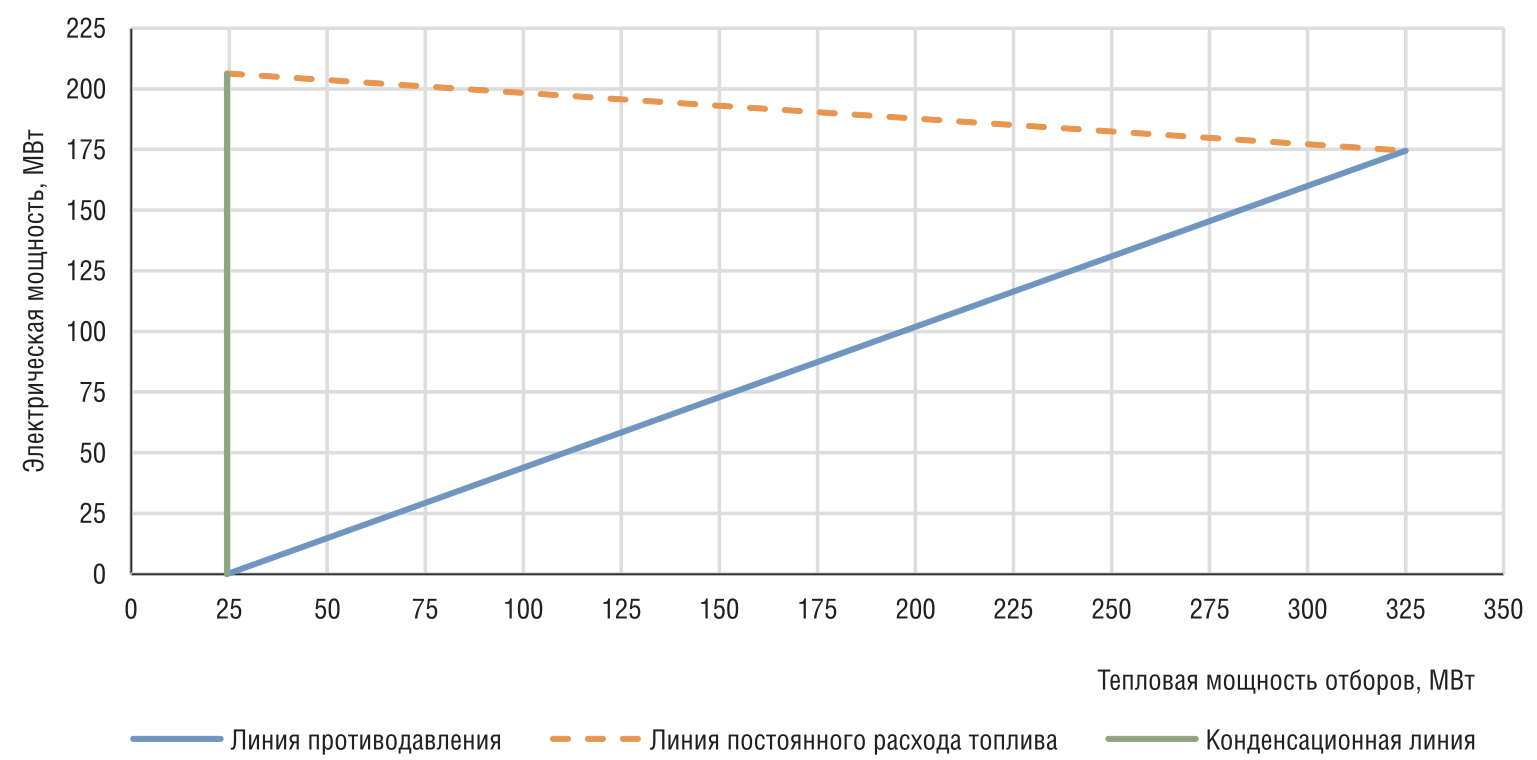

Рис. 5. Область работы турбины Т-175/210-130

Fig. 5. Area of operation of the turbine T-175/210-130

Область работы турбины, построенная с использованием энергетической характеристики, аналогична таковой для подхода, используемого в [1]. Отличие состоит в сдвиге линии работы в конденсационном режиме вправо, который вызван учетом холостого хода турбины. Существенным уточнением является также возможность учета зависимости эффективности работы турбины от ее мощности в отличие от методологии [1], где используется только номинальная эффективность. На рис. 6 показаны полные КПД работы турбины на так называемой линии

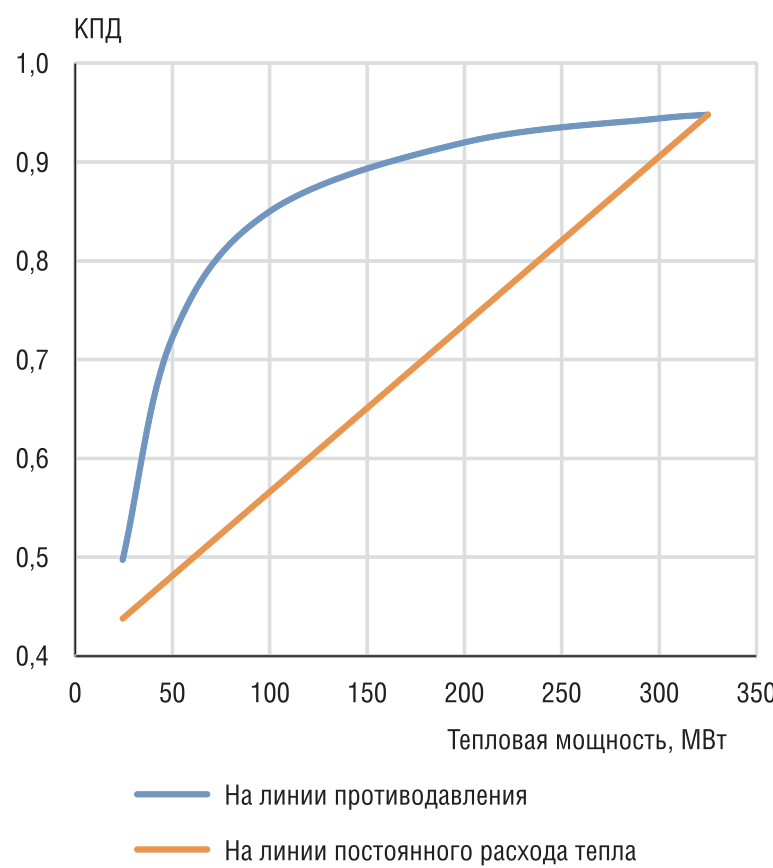

Рис. 6. Полный КПД на границах области работы турбины

Fig. 6. Total efficiency at the boundaries of the turbine work area противодавления (нижняя граница области работы на рис. 5) и линии постоянного расхода тепла (пунктирная линия на рис. 5).

Как видно, режим противодавления является наиболее выгодным, что соответствует физическому смыслу и лишний раз подтверждает адекватность аналитических выражений для энергетической характеристики турбины типа Т.

Зависимость КПД от электрической мощности в конденсационном режиме работы отражена на рис. 7. Можно видеть, что данная зависимость является довольно сильной. При 40\%-ной нагрузке эффективность снижается по отношению к номинальной примерно на $23 \%$, что является достаточным основанием для учета зависимости при оптимизационных расчетах.

На рис. 8 показаны рассчитанные с использованием энергетической характеристики составляющие КПД турбины Т-175/210-130 на линии $N=0,333 Q_{h}^{h}+66,667$, лежащей между линиями противодавления и постоянного расхода тепла. 
Из рисунка видно, что хотя зависимости составляющих эффективности для рассмотренной линии соотношения тепловой и электрической мощности не столь сильны, как на линии противодавления, однако они достаточно велики для того, чтобы утверждать о необходимости их учета при оптимизационных расчетах энергосистем.

Аналогичным образом могут быть получены с учетом зависимости от уровня мощности и давления в отборах пара модели КПД турбоагрегатов с турбинами производственно-отопительного типа ПТ. Энергетические характеристики для ряда турбин этого типа приведены в [2].

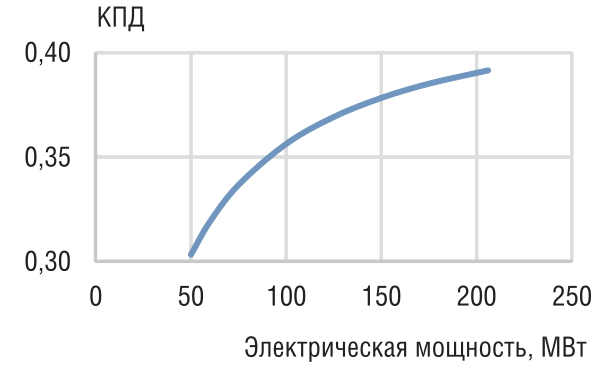

Рис. 7. Зависимость КПД от мощности в конденсационном режиме

Fig. 7. Efficiency versus power in condensation mode

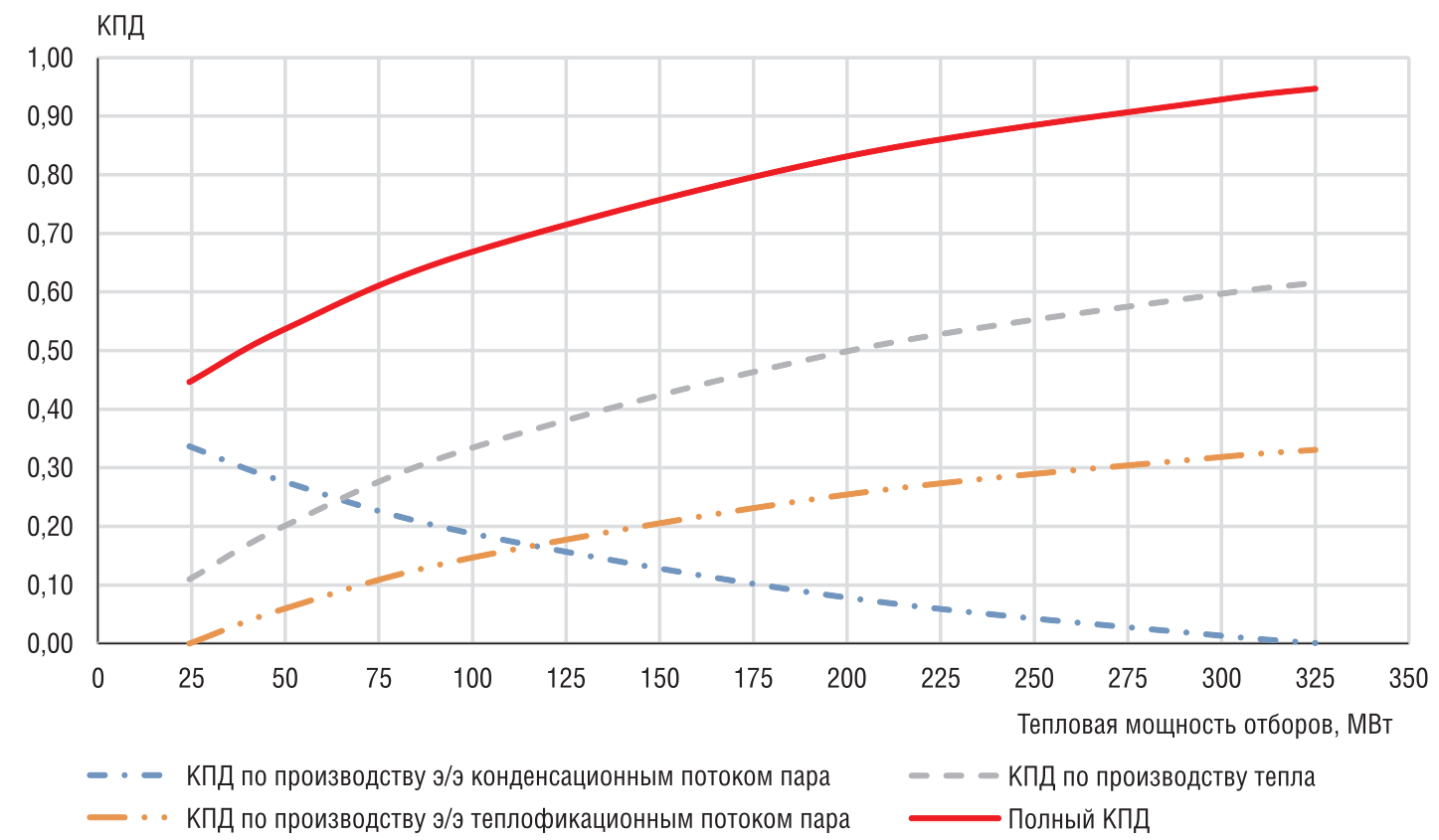

Рис. 8. Составляющие КПД турбины Т-175/210-130

Fig. 8. The components of the efficiency of the turbine T-175/210-130

Заключение. Для определения и оценки зависимости эффективности работы ряда турбоагрегатов конденсационного и теплофикационного типа от таких переменных параметров, как электрическая, тепловая мощность и давление пара в отборах турбины, использованы аналитические выражения энергетических характеристик.

Установлено, что для больших конденсационных турбин типа К-300-240 при мощности 50 \% от номинальной КПД меньше номинального более чем на $7 \%$ относительных (по отношению к КПД на номинальной мощности), а при мощности $30 \%$ от номинала - на 13,5 \%.

Для турбин противодавления типа Р-50-130/13 уменьшение противодавления на 14 \% в области номинального значения приводит к увеличению электрической мощности примерно на $8 \%$. Отсутствие учета наличия холостого хода приводит для данной турбины к значительному завышению электрической мощности, которую можно получить при определенной тепловой мощности. При тепловой мощности около $40 \%$ от номинальной превышение составит величину около $50 \%$ от более корректного значения, полученного с использованием энергетической характеристики.

Для теплофикационных турбин типа Т-175/210-130 при 40\%-ной нагрузке в конденсационном режиме работы эффективность снижается по отношению к номинальной примерно на $23 \%$.

Перечисленные обстоятельства дают основание сделать вывод о целесообразности использования достаточно простого алгоритма расчета энергетических характеристик для определения эффективности работы турбоагрегатов в программах, предназначенных для оптимизации энергетических систем. 


\section{Список использованных источников}

1. MESSAGE. Model for Energy Supply Strategy Alternatives and their General Environmental Impacts. User Manual. (DRAFT). International Atomic Energy Agency, 2007.

2. Качан, А. Д. Режимы работы и эксплуатации тепловых электрических станций / А. Д. Качан. - Минск: Выш. шк., 1978. - 146 с

3. Рыжкин, В.Я. Тепловые электрические станции: учеб. для вузов / В.Я. Рыжкин. - М.: Энергоатомиздат, 1987. $-328 \mathrm{c.}$

4. Трухний,А.Д. Теплофикационные паровые турбины и турбоустановки: учеб. пособие для вузов / А. Д. Трухний, Б. В. Ломакин. - М.: МЭИ, 2002. - 540 с.

\section{References}

1. International Atomic Energy Agency. MESSAGE. Model for Energy Supply Strategy Alternatives and their General Environmental Impacts. User Manual. Vienna, 2007.

2. Kachan A. D. Modes of Operation and Operation of Thermal Power Plants. Minsk, Vysheishaya shkola Publ., 1978. 146 p. (in Russian).

3. Ryzhkin V. Ya. Thermal Power Stations. Moscow, Energoatomizdat Publ., 1987. 328 p. (in Russian).

4. Truxnii A. D., Lomakin B. V. Heating Steam Turbines and Turbine Units. Moscow, Moscow Energy Institute, 2002. 540 p. (in Russian).

\section{Информация об авторах}

Мельников Алексей Сергеевич - аспирант, младший научный сотрудник, Объединенный институт энергетических и ядерных исследований - Сосны Национальной академии наук Беларуси (а/я 119, 220109, Минск, Республика Беларусь). E-mail: melnikov1991@bk.ru

Попов Борис Игоревич - кандидат технических наук, ведущий научный сотрудник, Институт энергетики Национальной академии наук Беларуси (ул. Академическая, 15, корп. 2, 220072, Минск, Республика Беларусь). E-mail: bipopovby@gmail.com

\section{Information about the authors}

Alexey S. Melnikov - Ph. D. Student, Junior Researcher, Joint Institute for Power and Nuclear Research - Sosny of the National Academy of Science of Belarus (P.O. box 119, 220109, Minsk, Republic of Belarus). E-mail: melnikov1991@bk.ru

Boris I. Popov - Ph. D. (Engineering), Leading Researcher, Institute of Energy of the National Academy of Sciences of Belarus (b. 2, 15, Akademicheskaya Str., 220072, Minsk, Republic of Belarus). E-mail: bipopovby@, gmail.com 